\title{
Impacto del método de presentación y de la lengua materna en la adquisición de vocabulario en español L2
}

\author{
Matías Hidalgo Gallardo - Università degli Studi di Bergamo \\ matiashidalgogallardo@gmail.com
}

Rebut / Received: 16/01/20

Acceptat / Accepted: 06/02/20

\begin{abstract}
Resumen. Impacto del método de presentación y de la lengua materna en la adquisición de vocabulario en español L2. La escasez de investigación empírica en el aprendizaje del léxico en español L2 ha motivado que en este trabajo se analice el impacto a corto y medio plazo del método de presentación léxica y de la lengua materna en la adquisición de vocabulario. En una muestra de 146 estudiantes hablantes nativos de italiano y de chino mandarín se han evaluado los efectos de tres métodos intencionales de presentación (lista, oraciones y texto glosado) y uno incidental (inferencia por contexto) en el conocimiento productivo y receptivo de la forma y del significado de una serie de ítems léxicos. Los resultados revelan en general ganancias estadísticamente significativas mayores para el aprendizaje intencional, con protagonismo del texto glosado; menor dificultad del conocimiento receptivo semántico; más eficacia del uso de oraciones para los sinohablantes y del uso del texto con glosas para los italoparlantes; y mayor proclividad al olvido mediante el empleo de listas.
\end{abstract}

Palabras clave: presentación del léxico, adquisición del vocabulario, italoparlantes, sinohablantes, español L2.

Abstract. Impact of the method of presentation and mother tongue on Spanish L2 vocabulary acquisition. The lack of empirical research regarding Spanish L2 vocabulary learning has motivated to analyse in this study the short-term and medium-term impact of the method of vocabulary presentation and of learners' mother tongue on vocabulary acquisition. The effects of three intentional methods of presentation (list, sentences and glossed text) and one incidental method (guessing from context) on form and meaning recall and recognition of a set of lexical items were assessed in a sample of 146 native Italian and Mandarin speakers. General results revealed statistically significant greater gains for intentional learning, especially through the glossed text-method. Receptive knowledge of meaning was more easily acquired. The sentence- 
method was more efficient for Mandarin speakers, whereas the glossed text-method was more convenient for Italian speakers. List-method was more prone to vocabulary oblivion.

Keywords: vocabulary presentation, vocabulary acquisition, Italian speakers, Chinese speakers, Spanish L2.

\section{Introducción}

Aunque vivimos un momento de revitalización léxica en la enseñanza de lenguas extranjeras, en español L2 carecemos de suficiente investigación empírica que respalde la didáctica del vocabulario. Nos guiamos por lo que se ha hecho para el inglés — sin profundizar en las posibles diferencias derivadas de la naturaleza lingüística-, por un relativo sentido común o seguimos tendencias, modas o mitos. Si bien contamos con trabajos que abordan los efectos de distintos métodos de trabajo (Barcroft, 2004, 2006, 2009), distintos tipos de actividades (San Mateo-Valdehíta, 2012; San Mateo-Valdehíta \& Chacón-García, 2019) o distintos tipos de aprendizaje (Agustín-Llach, 2009), incluso sobre la variación acústica en la presentación léxica (Barcroft \& Sommers, 2005), apenas tenemos datos específicos sobre el cotejo de métodos de presentación intencionales e incidentales en función del grado de contextualización. Ante esta realidad, pretendemos responder a las siguientes preguntas de investigación:

1. ¿Es mejor una presentación intencional o incidental del léxico?

2. ¿Cómo debemos presentar el vocabulario para aprenderlo más eficazmente: con o sin contexto?

3. ¿Habrá diferencias de aprendizaje según la lengua materna (LM) de los estudiantes?

A tal fin, analizaremos los efectos en el aprendizaje a corto y medio plazo de cuatro métodos receptivos de presentación léxica con explicación en español: tres métodos intencionales con distintos tipos de contexto (lista, oración y texto glosado) y uno incidental (inferencia por contexto). Para ello se evaluarán cuatro constructos: recuperación y reconocimiento de la forma y del significado. Incorporamos, además, el posible impacto de la LM de los informantes (italiano o mandarín), pues, además de las diferencias contextuales en la enseñanza de lenguas, la distancia lingüística podría influir en el aprendizaje (Ainciburu, 2017). De hecho, Swan (1997), ante esto último — sin obviar la distancia cultural—, expresa que:

Related languages often share a great deal of cognate vocabulary, and even where vocabulary is not cognate, there tend to be close translation equivalents: this can give learners an enormous advantage. Where languages have less common ground, word 
forms will generally be quite different; more information about word meaning and use also has to be acquired from scratch. (p. 6)

Con todo, asumimos que los métodos explícitos serán más efectivos (Hulstijn y Laufer, 2001; Laufer, 2006); que se observarán mejores resultados cuanto mayor sea el contexto ofrecido, al implicar mayor complejidad (Robinson, 2005) y procesamiento (Hulstijn \& Laufer, 2001), que el conocimiento receptivo será más fácilmente adquirido, al suponer un paso previo al productivo (Laufer, Elder, Hill \& Congdon, 2004); y que el conocimiento semántico será más asequible que el formal por ser considerado este último como el verdadero conocimiento productivo (Laufer et al., 2004) y porque, al no existir elaboración formal en los tratamientos recibidos, dirigir el procesamiento al componente semántico producirá un detrimento en el aprendizaje formal (Barcroft, 2002, 2012). Además, esperamos que la submuestra italiana, debido a la mencionada cercanía lingüística (Swan, 1997), obtenga mejores rendimientos que el subconjunto chino. Por último, presumimos que el método de listado será más eficaz para el grupo sinohablante, ya que este es un elemento de gran calado en su tradición educativa (Sánchez, 2008).

\section{Estado de la cuestión}

El debate sobre la instrucción incidental o intencional del vocabulario cuenta con detractores y defensores. Los partidarios del primer tipo, apoyados en la hipótesis del input (Krashen, 1985), defienden una enseñanza indirecta, fundamentalmente a través de la lectura, porque permite un aprendizaje más profundo (Nation, 1990), supera los límites temporales del aula (Schmitt, 2000) y está inserto en usos y fines comunicativos (Higueras, 2004). En cambio, los seguidores del segundo tipo alegan —en la línea del modelo de la atención a la forma (Long, 1991) — que el aprendizaje intencional previene inferencias erróneas del vocabulario (Kelly, 1990; Laufer, 1997; Paribakht \& Wesche, 1999) o que el léxico objeto de estudio pase inadvertido (Laufer \& Shmueli, 1997; Schmidt, 1993). En una comparación de ambas aproximaciones, Laufer (2006) y Barcroft (2009), entre otros, hallan que el aprendizaje intencional obtiene mejores resultados. Dada esta realidad dicotómica, varios autores abogan por una complementación de ambos enfoques (Higueras, 2004; Nation, 2001; Pigada \& Schmitt, 2006).

A la luz de lo expuesto, todo sugiere una aparente preeminencia de la instrucción intencional, dentro de la cual surge otro elemento de controversia: la contextualización/ descontextualización del vocabulario. Algunos autores (Crow, 1986) asocian la presentación sin contexto a un aprendizaje limitado a conectar la forma en L2 con el significado en L1, además de privar de un contexto que permita aprender el uso real de la palabra (Oxford \& Crookall, 1990), mientras que para otros estudiosos supone 
un método rápido y eficaz de adquirir gran cantidad de palabras (Nation, 2001). Por el contrario, a las tareas contextualizadas, al entrañar mayor complejidad (Robinson, 2005) y nivel de participación (Hulstijn \& Laufer, 2001), se les atribuye una mayor eficacia y gozan de un mayor respaldo.

En cuanto a la confrontación de distintos métodos de presentación, Seibert (1930) constató mejores resultados de retención léxica mediante el uso de listas que a través de oraciones. Griffin (1992) contempló diferencias en la eficacia de los métodos (listas y oraciones) en función del nivel de los aprendientes y de si la tarea era productiva o receptiva. Prince (1996) halló mayor memorización por medio de listas (con traducción en L1) que a través de contextos oracionales (con explicación en L2), aunque aquí las diferencias puedan vincularse a la lengua de instrucción. Laufer y Shmueli (1997) señalaron mayores ganancias de los métodos con atención a la forma (oraciones y lista) que de los más contextualizados (texto y texto adaptado), y en ambos casos superaron a la inferencia por contexto. Cabe señalar que, a diferencia de lo que ocurre en nuestro estudio, no se pidió a los informantes que trataran de recordar el vocabulario expuesto. Webb (2007), por su parte, no encontró diferencias significativas entre el empleo de listas y el de oraciones glosadas. Sin centrarse específicamente en la presentación, pues incluye tareas productivas, Agustín-Llach (2009) encuentra que el trabajo mediante listado y producción de oraciones obtuvo mejores resultados que la lectura, con y sin glosas, más preguntas de comprensión.

\section{Método}

\subsection{Muestra y materiales}

La intervención didáctica se realizó con 146 estudiantes universitarios de nivel intermedio de español, ${ }^{1}$ aunque en el postest se redujeron a 119 , puesto que no todos acudieron a clase el día de su realización. Ninguno conocía las palabras meta. Con edades comprendidas entre los 19 y los 22 años, 89 informantes hablan italiano como lengua materna y 57 , mandarín. En el postest, los sujetos se reducen a 66 italianos y 53 chinos. La recolección de datos tuvo lugar en el país de origen de cada grupo lingüístico: Italia y China. El tratamiento fue realizado separadamente para cada subconjunto por el investigador — que no es docente de ninguno de ellos - y cada submuestra lingüística fue dividida en cuatro grupos. En el caso italiano, los grupos se crearon aleatoriamente en el mismo momento del tratamiento, mientras que, en el caso chino, los grupos siguen la distribución por clases ya existente en su universidad. A cada grupo se le asignó aleatoriamente uno de los siguientes tratamientos de presentación léxica:

1. El nivel, B1-B2 según el Marco común europeo de referencia para las lenguas, fue determinado mediante la prueba de diagnóstico DIALANG (https://dialangweb.lancaster.ac.uk/). 
1. Lista. Las palabras meta aparecen en un listado en orden aleatorio con explicación en español. Duración: 10 minutos.

2. Oraciones. Las palabras, en orden aleatorio, son presentadas con ejemplos creados ad hoc, resaltadas y con explicaciones en español. Duración: 15 minutos.

3. Texto glosado. Las mismas palabras, resaltadas, forman parte de un texto creado exprofeso con glosas con explicaciones en español. Estas representan menos de un $5 \%$ del contenido del texto (304 palabras), por lo que se cumple con el principio de comprensibilidad de Hu y Nation (2000) según el cual un texto debe contener entre un $95-98 \%$ de léxico conocido para ser comprendido eficazmente. Un grupo de 48 estudiantes de perfil similar a la muestra confirmó previamente esta comprensibilidad. Duración: 20 minutos.

4. Inferencia por contexto. El grupo recibió el mismo texto que en la condición anterior, pero carente de toda explicación y resalte. Duración: 20 minutos.

Se evaluaron quince unidades monoverbales de muy baja frecuencia (Tabla 1), según el Corpus del Español (www.wordandphraseinfo/span/), sin relación entre sí y sin presencia de cognados que previamente, junto a otras quince palabras, fueron sometidas a evaluación por 76 estudiantes de nivel B2-C1 mediante una adaptación de The Vocabulary Knowledge Scale (Paribakht \& Wesche, 1993). Se seleccionaron las quince más desconocidas. Solo nueve formaron parte de la evaluación y las seis restantes sirvieron de distractores para mitigar una posible respuesta aleatoria, es decir, para prevenir que los informantes usaran todas las palabras indiscriminadamente.

TABla i. Listado DE PALABRAS META

\begin{tabular}{|l|l|l|}
\hline \multicolumn{3}{|c|}{ Palabras } \\
\hline ajar & cerote & fisgonear \\
\hline algaraza & chochear & jeta \\
\hline amente & descojonarse & sonsolillo \\
\hline cachas & enteco & tute \\
\hline carca & espichar & vacile \\
\hline
\end{tabular}

La evaluación del aprendizaje consistió en responder a dos pruebas sin anunciar: un test inmediato y un postest después de una semana. Se analizaron cuatro constructos en el siguiente orden: 
1. Recuperación de la forma: se medía el conocimiento productivo formal y se pidió a los informantes que escribieran las palabras cuyo significado encajara en el hueco que aparecía en un breve contexto.

2. Recuperación del significado: para computar el conocimiento productivo semántico, los participantes debían explicar (preferiblemente en español) el significado de la palabra meta en cuestión que estaba incluida en un pequeño contexto que no permitía inferir el significado a través del mismo.

3. Reconocimiento de la forma: se medía el conocimiento receptivo formal. A partir de un cuadro con las quince palabras meta, los estudiantes debían completar el hueco que aparecía en un breve contexto.

4. Reconocimiento del significado: para medir el conocimiento receptivo semántico, los sujetos debían elegir entre cuatro opciones la que mejor explicara el significado de la palabra meta incorporada en una oración.

El orden de las pruebas fue cuidadosamente secuenciado, evaluando primero el conocimiento productivo y después el receptivo, para prevenir un efecto aprendizaje (Webb, 2005, 2007). Cada constructo se componía de nueve ítems (ajar, algaraza, carca, cerote, enteco, espichar, fisgonear, jeta y sonsolillo) y cada acierto sumaba un punto sin penalización por respuestas erróneas o no contestadas. El orden de los ítems fue alterado entre los distintos constructos y entre las pruebas para prevenir un efecto recuerdo.

\subsection{Procedimiento}

Una prueba piloto con 48 estudiantes de nivel B1-B2 permitió precisar las instrucciones, confirmar la adecuación de las palabras meta y del texto y determinar los tiempos de administración.

Tras esto se administró un cuestionario sociolingüístico para definir el perfil del informante y valorar si alguno conocía el vocabulario meta con una adaptación del Eurocentres Vocabulary Size Test (Meara \& Jones, 1990), en cuyo supuesto el informante fue descartado de la investigación.

Después se administraron los tratamientos individualmente, sin usar dispositivos de consulta y sin poder tomar notas. En los métodos directos los estudiantes debían tratar de recordar las palabras, pero no en el indirecto. Inmediatamente después se cumplimentó el test y una semana después, el postest.

\section{Resultados}

Exponemos los resultados cuantitativos a corto y medio plazo según el método de presentación y el constructo analizado. Además, se compara la evolución temporal de la retención léxica según los datos de los sujetos que participaron en ambas pruebas. 


\subsection{Resultados del test}

\subsubsection{Resultados del test por método de presentación}

Con un acierto promedio general del $57,9 \%$, los aciertos totales (variable dependiente) según el método de presentación (variable independiente) revelan un rendimiento superior del método de Texto glosado, seguido del de Oraciones y de la presentación en listado (Tabla 2). En el extremo más bajo hallamos el método indirecto. Las desviaciones típicas muestran altos niveles de dispersión en las respuestas.

El análisis de varianza (ANOVA) de los datos, que siguen una distribución normal, determina que estas diferencias generales según el método de presentación son significativas $(F[3,142]=23,171, p=, 000)$. Las comparaciones múltiples post hoc (DHS de Tukey, al asumirse varianzas iguales) sitúan dichas diferencias exclusivamente entre el método inferencial — con menos aciertos—y el resto ( $p=, 000$ en todos los contrastes).

TABla 2. DesCriptivos ESTADíSTICOS SEGÚN EL MÉTODO DE PRESENTACIÓN Y LA LM (TEST)

\begin{tabular}{|c|c|c|c|c|}
\hline Método & LM & $\mathbf{N}$ & Media* & Desviación típica \\
\hline \multirow[t]{3}{*}{ Lista } & Italiano (IT) & 24 & 21,96 & 7,24 \\
\hline & Mandarín $(\mathrm{CH})$ & 16 & 22,44 & 6,77 \\
\hline & Subtotal & 40 & 22,15 & 6,97 \\
\hline \multirow[t]{3}{*}{ Oraciones } & Italiano & 24 & 21,63 & 9,81 \\
\hline & Mandarín & 12 & 27,33 & 4,85 \\
\hline & Subtotal & 36 & 23,50 & 8,84 \\
\hline \multirow[t]{3}{*}{ Texto glosado } & Italiano & 20 & 27,05 & 8,23 \\
\hline & Mandarín & 12 & 24,33 & 6,92 \\
\hline & Subtotal & 32 & 26,03 & 7,77 \\
\hline \multirow{3}{*}{$\begin{array}{l}\text { Inferencia por } \\
\text { contexto }\end{array}$} & Italiano & 21 & 14,14 & 5,28 \\
\hline & Mandarín & 17 & 10,59 & 6,08 \\
\hline & Subtotal & 38 & 12,6 & 5,85 \\
\hline \multirow[t]{2}{*}{ Total } & Italiano & 89 & 21,17 & 8,93 \\
\hline & Mandarín & 57 & 20,33 & 9,00 \\
\hline & & 146 & 20,84 & 8,93 \\
\hline
\end{tabular}


En función de la LM, los italianos recuperan y reconocen más palabras independientemente del método de presentación. Si ahondamos en el tipo de tratamiento recibido, los sinohablantes sobresalen en el uso de oraciones y los italianos en el texto glosado. La ANOVA factorial univariante $(F[3,138]=2,741, p=, 046)$, mediante las comparaciones por pares, revela que estas diferencias solo son significativas para el mayor rendimiento chino en el método de Oraciones $(p=, 028)$.

\subsubsection{Resultados del test por método y constructo}

Independientemente del método de presentación, constatamos que las puntuaciones más altas se vinculan al conocimiento semántico (tanto receptivo como productivo), con el reconocimiento formal muy próximo, mientras que la tarea de recuperación formal presenta una puntuación notablemente menor, lo que la hace la más compleja (Tabla 3).

Si profundizamos en los efectos de los métodos de presentación, se confirma que el Texto glosado destaca en todas las dimensiones, mientras que la Inferencia otorga peores resultados; con una mayor facilidad para la adquisición del conocimiento semántico a través del texto con glosas, pero del conocimiento receptivo mediante la deducción por contexto. En el resto de tratamientos, las Oraciones sobrepasan a la Lista en todos los constructos, excepto en el reconocimiento semántico, con resultados muy próximos.

El análisis multivariante de varianza (MANOVA) revela una diferencia unísona significativa entre los métodos de presentación $(F[12,368]=8,104, p=, 000)$, diferencia que se observa también para cada constructo por separado. Así, en la recuperación formal $(F[3,142]=12,850, p=, 000)$, las comparaciones post hoc (Games-Howell, al asumirse varianzas desiguales) localizan dichas diferencias significativas solo en los contrastes con la Inferencia por contexto ( $p=, 000$ en comparación con los demás métodos). Para el resto de constructos $(F[3,142]=32,395, p=, 000$ para la producción semántica, $F[3,142]=9$, $428, p=, 000$ para el reconocimiento de la forma y $F[3,142]=20,719, p=, 000$ para la comprensión del significado), según el DHS de Tukey, las diferencias son significativas también entre el método indirecto y el resto $(p=, 000$ en todos los contrastes de la prueba de recuperación semántica; $p=, 035, p=, 001$ y $p=, 000$, respectivamente, en comparación con el listado, las oraciones y el texto glosado en la medición del reconocimiento formal, y $p=, 000$ en todos los cotejos dentro del reconocimiento semántico).

Tabla 3. Descriptivos estadísticos por mÉtodo, LM y constructo (Test)

\begin{tabular}{|l|l|c|c|c|c|c|c|c|c|c|}
\hline & & & \multicolumn{2}{|c|}{ RpF } & \multicolumn{2}{c|}{ RpS } & \multicolumn{2}{c|}{ RmF } & \multicolumn{2}{c|}{ RmS } \\
\hline \multirow{2}{*}{ Método } & $\mathbf{L M}$ & $\mathbf{N}$ & $\mathbf{M}^{*}$ & $\mathbf{D T}$ & $\mathbf{M}$ & $\mathbf{D T}$ & $\mathbf{M}$ & $\mathbf{D T}$ & $\mathbf{M}$ & DT \\
\hline \multirow{3}{*}{ Lista } & IT & 24 & 3,63 & 2,28 & 6,17 & 2,46 & 4,92 & 2,00 & 7,25 & 1,75 \\
\cline { 2 - 11 } & CH & 16 & 3,50 & 2,25 & 5,94 & 2,29 & 5,06 & 2,29 & 7,94 & 1,24 \\
\cline { 2 - 11 } & Subtotal & 40 & 3,58 & 2,24 & 6,08 & 2,37 & 4,98 & 2,09 & 7,53 & 1,59 \\
\hline
\end{tabular}




\begin{tabular}{|c|c|c|c|c|c|c|c|c|c|c|}
\hline \multirow[t]{3}{*}{ Oraciones } & IT & 24 & 3,75 & 2,97 & 5,79 & 2,48 & 5,21 & 2,70 & 6,88 & 2,47 \\
\hline & $\mathrm{CH}$ & 12 & 4,33 & 2,27 & 7,75 & 1,29 & 6,50 & 2,02 & 8,75 &, 45 \\
\hline & Subtotal & 36 & 3,94 & 2,74 & 6,44 & 2,34 & 5,64 & 2,54 & 7,50 & 2,21 \\
\hline \multirow[t]{3}{*}{ Texto } & IT & 20 & 5,10 & 2,99 & 6,85 & 2,13 & 7,10 & 2,34 & 8,00 & 1,62 \\
\hline & $\mathrm{CH}$ & 12 & 3,83 & 3,22 & 6,92 & 1,73 & 5,00 & 2,73 & 8,58 & ,67 \\
\hline & Subtotal & 32 & 4,63 & 3,09 & 6,88 & 1,96 & 6,31 & 2,66 & 8,22 & 1,36 \\
\hline \multirow[t]{3}{*}{ Inferencia } & IT & 21 & 1,62 & 1,47 & 3,05 & 1,53 & 3,95 & 1,75 & 5,52 & 1,63 \\
\hline & $\mathrm{CH}$ & 17 & 0,88 & 1,54 & 1,82 & 1,98 & 3,06 & 1,98 & 4,82 & 1,70 \\
\hline & Subtotal & 38 & 1,29 & 1,52 & 2,50 & 1,83 & 3,55 & 1,88 & 5,21 & 1,68 \\
\hline \multirow[t]{2}{*}{ Total } & IT & 89 & 3,52 & 2,74 & 5,48 & 2,59 & 5,26 & 2,46 & 6,91 & 2,08 \\
\hline & $\mathrm{CH}$ & 57 & 2,96 & 2,65 & 5,30 & 3,01 & 4,75 & 2,52 & 7,32 & 2,04 \\
\hline & & 146 & 3,30 & 2,71 & 5,41 & 2,75 & 5,06 & 2,49 & 7,07 & 2,07 \\
\hline
\end{tabular}

*Puntuación máxima $=9$

La realidad general observada anteriormente — supremacía del conocimiento semánticose corrobora en función de la LM, con más aciertos de la muestra italófona en todos los constructos, salvo en el reconocimiento semántico. El análisis de varianza univariante señala que las diferencias en función de la lengua no son significativas para la recuperación formal $(F[3,138]=, 850, p=, 469)$, pero sí para el resto de constructos: en la evaluación de la recuperación semántica $(F[3,138]=3,417, p=, 019)$ se concretan en el método de Oraciones $(p=, 009)$; en la medición del reconocimiento formal $(F[3,138]=3,294, p=, 022)$ se observan en el método de Texto glosado $(p=, 011)$, y en el reconocimiento semántico $(F[3,138]=3,402, p=, 020)$ se aprecian, nuevamente, en el método de ejemplos $(p=, 002)$.

\subsection{Resultados del postest}

\subsubsection{Resultados del postest por método de presentación}

Se confirma la supremacía del método de Texto glosado y el menor rendimiento del método de Inferencia. El método de Oraciones sigue ocupando la segunda posición, seguido del método de Lista. La heterogeneidad en las respuestas, indicada por la desviación típica, continúa siendo bastante alta (Tabla 4). La ANOVA $(F[3,115]=22,931, p=, 000)$ y las comparaciones múltiples post hoc (DHS de Tukey) confirman en este postest que los resultados obtenidos por los métodos de presentación intencional son significativamente mejores que los del método incidental ( $p=, 000$ en los contrastes entre dichos métodos).

Asimismo, constatamos que el recuerdo léxico decrece con el paso del tiempo, tanto en la media total como en función del método, con la única excepción del método de 
Inferencia, cuyo promedio aumenta. La ANOVAde medidas repetidas $(F[3,115]=9,829$, $p=, 000)$ nos permite analizar las diferencias temporales y afirmar que el empeoramiento de la calidad del recuerdo es significativo para los métodos directos ( $p=, 000$ para el listado, $p=, 001$ para los ejemplos y $p=, 001$ para el texto con glosas), al igual que la mejora para el método indirecto $(p=, 009)$ (ver tabla 4 ).

En este postest, los italianos vuelven a superar a los chinos en los resultados generales, con una reseñable actuación de los primeros cuando reciben el léxico mediante lista, texto glosado e inferencia, pero no mediante las oraciones. La ANOVA factorial univariante $(F[3,111]=2,920, p=, 037)$ indica que solo los mejores resultados obtenidos por los italoparlantes en los métodos de Texto glosado $(p=, 007)$ y de Inferencia $(p=, 008)$ son estadísticamente significativos.

En todos los métodos se produce un decrecimiento de los resultados de ambos grupos lingüísticos — más acuciado en la muestra china—, salvo en la tarea de inferencia, donde los dos conjuntos mejoran, con un papel destacado de los italianos. Aunque la ANOVA de medidas repetidas $(F[3,111]=2,069 p=, 108)$ no testifica diferencias temporales significativas, las comparaciones por pares sí las señalan para ambos grupos en el descenso que se produce cuando se presenta el léxico con lista $(p=, 002$ y $p=, 007$, respectivamente), exclusivamente para los chinos en el decrecimiento en los métodos de Oraciones $(p=, 000)$ y de Texto glosado $(p=, 000)$ y únicamente para los italianos en el incremento en la deducción por contexto $(p=, 000)$.

Tabla 4. Descriptivos estadísticos Según el método de presentación y la LM (postest)

\begin{tabular}{|l|l|c|c|c|}
\hline \multicolumn{1}{|c|}{ Método } & \multicolumn{1}{|c|}{ LM } & N & Media $^{*}$ & Desviación típica \\
\hline \multirow{4}{*}{ Lista } & Italiano & 21 & 19,76 & 7,86 \\
\cline { 2 - 5 } & Mandarín & 14 & 19,71 & 7,13 \\
\cline { 2 - 5 } & Subtotal & 35 & 19,74 & 7,47 \\
\hline \multirow{4}{*}{ Oraciones } & Italiano & 12 & 20,08 & 9,93 \\
\cline { 2 - 5 } & Mandarín & 12 & 21,67 & 6,56 \\
\cline { 2 - 5 } & Subtotal & 24 & 20,88 & 8,27 \\
\hline \multirow{3}{*}{ Texto glosado } & Italiano & 17 & 26,41 & 7,72 \\
\cline { 2 - 5 } & Mandarín & 12 & 18,67 & 8,70 \\
\cline { 2 - 5 } & Subtotal & 29 & 23,21 & 8,88 \\
\hline \multirow{3}{*}{$\begin{array}{l}\text { Inferencia por } \\
\text { contexto }\end{array}$} & Italiano & 16 & 18,06 & 6,62 \\
\cline { 2 - 5 } & Mandarín & 15 & 10,73 & 5,22 \\
\cline { 2 - 5 } & Subtotal & 31 & 14,52 & 6,96 \\
\hline
\end{tabular}




\begin{tabular}{|l|l|c|c|c|}
\hline \multirow{2}{*}{ Total } & Italiano & 66 & 21,12 & 8,42 \\
\cline { 2 - 5 } & Mandarín & 53 & 17,38 & 8,00 \\
\hline & 119 & 19,45 & 8,41 \\
\hline
\end{tabular}

\subsubsection{Resultados del postest por método y constructo}

Tras el postest, los resultados generales para cada dimensión (Tabla 5) ratifican que, sin considerar la forma de presentación, el conocimiento receptivo es más asequible que el productivo, algo que no ocurría tan claramente en el test inmediato. El conocimiento productivo formal continúa siendo el más complejo.

Los resultados por método y constructo corroboran los datos del test: la exposición mediante texto glosado arroja mayores beneficios en todas las dimensiones; los informantes que trabajan con el método indirecto son los más rezagados y el trabajo con ejemplos supera al listado en todos los constructos menos en el del reconocimiento semántico, aunque con datos muy cercanos entre ellos.

$\operatorname{LaMANOVA}(F[12,296,6]=4,344, p=, 000)$ indica que las diferencias en los promedios por método son significativas. Para la recuperación de la forma $(F[3,115]=3,013$, $p=, 033)$, el DHS de Tukey — aplicable también en los siguientes constructos- muestra que las únicas diferencias significativas se producen entre el método de Inferencia, con los resultados más bajos, y el método de Texto glosado, con los mejores rendimientos ( $p=, 032$ ). En la recuperación semántica $F[3,115]=7,476, p=, 000)$ estas diferencias se extienden a todos los métodos en comparación con el incidental ( $p=, 017$ con respecto al uso de la lista, $p=, 003$ en contraste con los ejemplos y $p=, 000$ si lo cotejamos con el texto glosado). En la evaluación del reconocimiento formal $F(3,115)=2,818, p=, 042$ de nuevo solo hay diferencias significativas entre el método inferencial y el método textual $(p=, 027)$. La dimensión del reconocimiento del significado $F(3,115)=14,432, p=, 000$ vuelve a manifestar claras diferencias significativas entre el método indirecto de presentación y los métodos directos ( $p=, 000$ en todos estos contrastes).

TABla 5. Descriptivos estadísticos POR MÉtodo, LM y CONSTRUCto (POSTEST)

\begin{tabular}{|c|c|c|c|c|c|c|c|c|c|c|}
\hline \multirow[b]{2}{*}{ Método } & \multirow[b]{2}{*}{$\mathbf{L M}$} & \multirow[b]{2}{*}{$\mathbf{N}$} & \multicolumn{2}{|l|}{$\mathrm{RpF}$} & \multicolumn{2}{|l|}{$\mathrm{RpS}$} & \multicolumn{2}{|l|}{$\mathrm{RmF}$} & \multicolumn{2}{|l|}{$\mathrm{RmS}$} \\
\hline & & & $\mathbf{M}^{*}$ & DT & $\mathbf{M}$ & DT & $\mathbf{M}$ & DT & $\mathbf{M}$ & DT \\
\hline \multirow[t]{3}{*}{ Lista } & IT & 21 & 2,05 & 2,67 & 4,86 & 2,69 & 5,19 & 2,64 & 7,67 & 1,28 \\
\hline & $\mathrm{CH}$ & 14 & 1,43 & 2,31 & 5,29 & 2,40 & 5,21 & 2,49 & 7,79 & 1,31 \\
\hline & Subtotal & 35 & 1,80 & 2,52 & 5,03 & 2,55 & 5,20 & 2,54 & 7,71 & 1,27 \\
\hline
\end{tabular}




\begin{tabular}{|c|c|c|c|c|c|c|c|c|c|c|}
\hline \multirow[t]{3}{*}{ Oraciones } & IT & 12 & 3,00 & 3,19 & 4,83 & 2,62 & 5,33 & 2,87 & 6,92 & 2,02 \\
\hline & $\mathrm{CH}$ & 12 & 1,42 & 2,78 & 6,33 & 2,02 & 5,58 & 2,64 & 8,33 & 0,99 \\
\hline & Subtotal & 24 & 2,21 & 3,04 & 5,58 & 2,41 & 5,46 & 2,70 & 7,63 & 1,72 \\
\hline \multirow[t]{3}{*}{ Texto } & IT & 17 & 4,41 & 3,00 & 6,88 & 2,09 & 7,06 & 2,38 & 8,06 & 1,25 \\
\hline & $\mathrm{CH}$ & 12 & 2,08 & 2,75 & 4,67 & 2,39 & 4,67 & 3,11 & 7,25 & 1,60 \\
\hline & Subtotal & 29 & 3,45 & 3,08 & 5,97 & 2,44 & 6,07 & 2,92 & 7,72 & 1,44 \\
\hline \multirow[t]{3}{*}{ Inferencia } & IT & 16 & 2,44 & 2,56 & 4,25 & 1,98 & 5,13 & 2,09 & 6,25 & 1,44 \\
\hline & $\mathrm{CH}$ & 15 & 0,53 & 0,92 & 2,20 & 1,78 & 3,07 & 2,37 & 4,93 & 1,67 \\
\hline & Subtotal & 31 & 1,52 & 2,14 & 3,26 & 2,13 & 4,13 & 2,43 & 5,61 & 1,67 \\
\hline \multirow[t]{2}{*}{ Total } & IT & 66 & 2,92 & 2,92 & 5,23 & 2,53 & 5,68 & 2,57 & 7,29 & 1,60 \\
\hline & $\mathrm{CH}$ & 53 & 1,32 & 2,26 & 4,51 & 2,61 & 4,57 & 2,76 & 6,98 & 1,94 \\
\hline & 119 & 2,21 & 2,76 & 4,91 & 2,58 & 5,18 & 2,70 & 7,15 & 1,76 \\
\hline
\end{tabular}

En comparación con el test, los informantes muestran rendimientos más bajos tras este lapso en la recuperación de la forma y del significado, pero mejoran sus resultados — muy superficialmente - en el reconocimiento formal y semántico. Asimismo, se evidencia un olvido generalizado para todos los tratamientos, salvo para el método de Inferencia, que incrementa sus promedios en todos los constructos. También encontramos mejoras en el grupo del método de Lista - bastante leves - para el reconocimiento formal y semántico y en el que trabaja con ejemplos en el reconocimiento del significado.

En este contraste temporal, la ANOVA de medidas repetidas expone diferencias significativas para la recuperación formal $(F[3,115]=6,005, p=, 001)$ cuando intervienen los métodos directos, que acusan más la desmemoria: $p=, 000$ para la lista y las oraciones y $p=, 001$ para el texto glosado. La mejora de resultados del método inferencial no es significativa $(p=, 525)$. También hallamos diferencias significativas en la prueba de recuperación semántica $F(3,115)=8,370, p=, 000$, que afecta tanto a la disminución de los puntajes de los métodos de Lista $(p=, 000)$, Oraciones $(p=, 003)$ y Texto glosado $(p=, 007)$ como al incremento del método indirecto $(p=, 019)$. En el contraste diacrónico para el reconocimiento de la forma $F(3,115)=2,931, p=, 037$ la significatividad se restringe al aumento en el método de Inferencia $(p=, 016)$. En la evaluación del conocimiento receptivo del significado $F(3,115)=2,972, p=, 035$ dichas diferencias son significativas para el olvido observado en el método de Texto glosado $(p=, 030)$ y para la mejora mediante la deducción por contexto $(p=, 049)$.

Tanto italianos — con resultados generales mejores - como chinos presentan mayor facilidad para el conocimiento receptivo (tanto semántico como formal) en el postest, a diferencia de lo que ocurre en el test, donde destacaba el conocimiento semántico (receptivo y productivo). La ANOVA univariante señala que no hay significatividad general para la 
recuperación formal $(F[3,111]=, 620, p=, 603)$, aunque sí la hay entre las diferencias por LM para el método intencional textual $(p=, 019)$. Sí existe significatividad en las diferencias lingüísticas para la recuperación semántica $(F[3,111]=4,495, p=, 005)$, concretamente en los métodos de Texto glosado $(p=, 011)$ y de Inferencia $(p=, 014)$, los mismos métodos en los que se aprecian las diferencias para el reconocimiento formal ( $p=, 015$ у $p=, 027$, respectivamente), pese a que los resultados generales de la ANOVA factorial univariante no se muestren significativamente diferentes $(F[3,111]=2,047$, $p=, 111)$. En la tarea semántica receptiva $(F[3,111]=4,557, p=, 005)$ estas diferencias son significativas dentro del método oracional $(p=, 018)$ y del método inferencial $(p=, 013)$.

Las diferencias temporales en función de la LM y del método observadas en la recuperación de la forma -que aparentemente no son significativas en general $(F(3,111)=, 854, p=, 467)$ — se concretan de modo significativo en el método de Lista y en el de Texto glosado para ambos conjuntos de hablantes $(p=, 001$ para italianos y chinos en el primer tratamiento y $p=, 040$ para italófonos у $p=, 007$ para sinófonos en el segundo), pero solo para los hablantes de chino en el método de Oraciones $(p=, 000)$. Sí son significativas las diferencias generales para la recuperación semántica $(F[3,111]=4$, 413, $p=, 006)$, que se sintetizan en el método de listado $(p=, 000)$ y de Inferencia $(p=, 009)$ para los italianos y en el método de ejemplos $(p=, 010)$ y de Texto glosado $(p=, 000)$ para los chinos. En la dimensión del reconocimiento formal, pese a una falta de significatividad temporal general $(F[3,111]=, 534, p=, 660)$, observamos diferencias significativas para los italianos en el método inferencial $(p=, 006)$. Lo mismo ocurre con el reconocimiento semántico $(F(3,111)=, 929, p=, 429)$, aunque en este caso se atisban para los chinos en el método textual $(p=, 000)$ y para los italianos en el método inferencial $(p=, 002)$.

\section{Discusión y conclusiones}

Los resultados generales esbozan un panorama en el que cuanto mayor es el contexto, más ganancias léxicas se observan a corto y a medio plazo, lo que parece contradecir los resultados de Laufer y Shmueli (1997) y de Agustín-Llach (2009). Sin embargo, los diferentes puntajes solo son estadísticamente significativos en el contraste entre los métodos directos y el método indirecto, lo que respalda nuestra conjetura de mayor eficacia del aprendizaje intencional, pero no así nuestra hipótesis de que cuanto más contexto, más beneficio, pues solo queda parcialmente confirmada a través de los promedios. La mayor conciencia y esfuerzo memorístico de la presentación intencional han podido contribuir a estos mejores resultados.

La evaluación inmediata refleja mayor facilidad en la adquisición del conocimiento semántico, con protagonismo del reconocimiento, mientras que en el peritaje postergado destaca la retención del conocimiento receptivo semántico y formal. En cambio, la tarea más compleja es la recuperación formal. Esto coincide con lo expuesto por Laufer 
et al. (2004) acerca de la mayor dificultad en la producción léxica y lo señalado por Webb (2005) sobre la predisposición a conseguir un conocimiento más receptivo si las tareas de aprendizaje — como es nuestro caso — son receptivas y, además, confirma otra de nuestras hipótesis. También parece confirmarse nuestra presunción sobre la mejor adquisición del conocimiento semántico.

Los promedios obtenidos por método y constructo en ambos espacios temporales ratifican la superioridad del texto glosado y la inferioridad de la deducción por contexto. De nuevo, la significatividad se localiza mayoritariamente en las diferencias entre los métodos intencionales y el incidental, diferencias que en el postest se concentran entre el método de Inferencia y el de Texto glosado para el conocimiento formal, lo que refuerza la idea de que el aprendizaje intencional favorece el apercibimiento del vocabulario, sobre todo en su plano formal.

Del cotejo diacrónico se desprende significativamente que el recuerdo léxico se deteriora con el paso del tiempo para todos los métodos salvo para el trabajo de inferencia por contexto, donde aumentan los promedios de acierto. Este deterioro, encabezado por el método de Lista — que parece ser más proclive al olvido- se aglutina en las tareas productivas, mientras que el aumento, capitaneado por la inferencia por contexto, afecta ligeramente a las tareas receptivas. Este incremento visto en el método inferencial puede achacarse a un efecto de aprendizaje propiciado por la propia evaluación, en la que las palabras meta aparecen frecuentemente.

En cuanto al rendimiento léxico general por LM, los italoparlantes superan a los sinohablantes en el test y en el postest, debido seguramente a la proximidad lingüística, aunque, por método, los segundos destacan cuando trabajan con los ejemplos $\mathrm{y}$, en menor medida, con la lista durante el test, pero solo con los ejemplos durante el postest. Además, solo se observan diferencias significativas en el método de Oraciones a corto plazo, a favor de los chinos, y a medio plazo en los métodos de Texto glosado y de Inferencia, en pro de los italianos, lo que refuta nuestra hipótesis de mayor eficacia del método de Lista para los sinohablantes. Es más, el uso de este método acusa más el olvido, especialmente en los hablantes de mandarín. Este método es el que menos propicia la creación de relaciones y redes léxico-semánticas, por lo que probablemente no favorece un aprendizaje significativo que permita realizar anclajes con otros elementos.

Un elemento reseñable en los resultados es la dispersión en las respuestas, lo que debe hacernos pensar en la influencia de los factores individuales de los informantes. Otras limitaciones dignas de investigación futura tienen que ver con la naturaleza del léxico o el impacto de la lengua de explicación del mismo. Además, en relación con la evaluación, habrá que indagar en los efectos de los métodos de presentación más allá de la conexión forma-significado, es decir, de índole sintáctica y gramatical (Webb, 2005, 2009).

De todo esto se desprende que, tal y como sostienen Nation (2001) o AgustínLlach (2009), la sola exposición al léxico no es suficiente; debe complementarse con el aprendizaje intencional. Por tal motivo, nuestro punto de partida en el aula debe ser la instrucción directa del vocabulario, que debe acompañarse con otros métodos 
indirectos como la lectura extensiva y/o enfocada, pues favorece la recurrencia y reciclaje del vocabulario. Asimismo, haciéndonos eco de las palabras de Higueras (2004, p. 16), "la metodología que adoptemos para la enseñanza del léxico debería ser consecuente con lo que sabemos sobre la forma en que se adquiere el léxico", de modo que, si hemos observado diferencias entre sinohablantes e italohablantes, lo lógico es que nos adaptemos metodológicamente a su forma de aprendizaje.

\section{Referencias}

Agustín-Llach, M. P. (2009). The effect of reading only, reading and comprehension, and sentence writing in lexical learning in a foreign language: some preliminary results. RESLA, 22, 9-33.

Ainciburu, M. C. (2017). La adquisición del vocabulario de las lenguas extranjeras a la luz de los hallazgos neurolingüísticos. En F. del Barrio (Ed.), Palabras, vocabulario, léxico: la lexicología aplicada a la didáctica y a la diacronia (pp. 35-48). Venecia: Università Ca' Foscari.

Barcroft, J. (2002). Semantic and structural elaboration in L2 Lexical acquisition. Language Learning, 52(2), 323-363.

Barcroft, J. (2004). Effects of sentence writing in second language lexical acquisition. Second Language Research, 20(4), 303-334.

Barcroft, J. (2006). Can Writing a new word detract from Learning it? More negative effects of forced output during vocabulary learning. Second Language Research, 22(4), 487-497.

Barcroft, J. (2009). Effects of synonym generation on incidental and intentional vocabulary learning during second language reading. TESOL Quarterly, 43(1), 79103.

Barcroft, J. (2012). Input-based incremental vocabulary instruction. Alexandria, VA: TESOL International Association.

Barcroft, J., y Sommers, M. (2005). Effects of acoustic variability on second language vocabulary learning. Studies in Second Language Acquisition, 27(3), 387-414.

Crow, J. T. (1986). Receptive vocabulary acquisition for reading comprehension. The Modern Language Journal, 70(3), 242-250.

Griffin, G. F. (1992). Aspects of the psychology of second language vocabulary list learning (Tesis doctoral), University of Warwick.

Higueras, M. (2004). Claves prácticas para la enseñanza del léxico. Revista Nebrija de Lingüistica Aplicada a la Enseñanza de Lenguas, 9, 172-192.

Hu, M., \& Nation, P. (2000). Vocabulary density and reading comprehension. Reading in a Foreign Language, 13(1), 403-430.

Hulstijn, J. H., \& Laufer, B. (2001). Some Empirical Evidence for the Involvement Load Hypothesis in Vocabulary acquisition. Language Learning, 51(3), 539-558. 
Kelly, P. (1990). Guessing: No substitute for systematic learning of lexis. System, 18(2), 199-207.

Krashen, S. D. (1985). The input hypothesis: Issues and implications. Nueva York: Longman.

Laufer, B. (1997). The lexical plight in second language reading: Words you don't know, words you think you know and words you can't guess. En J. Coady y T. Huckin (Eds.), Second language vocabulary acquisition: A rationale for pedagogy (pp. 20-34). Cambridge: Cambridge University Press.

Laufer, B. (2006). Comparing focus on form and focus on formS in second-language vocabulary learning. The Canadican Modern Language Review, 63(4), 149-166.

Laufer, B., \& Shmueli, K. (1997). Memorizing new words: Does teaching have anything to do with it? RELC Journal, 28, 89-108.

Laufer, B., Elder, C., Hill, K., \& Congdon, P. (2004). Size and strength: Do we need both to measure vocabulary knowledge? Language Testing, 21(2), 202-226.

Long, M. (1991). Focus on form: A design feature in language teaching methodology. En K. de Bot, R. Ginsberg \& C. Kramsch (Eds.), Foreign language research in crosscultural perspective (pp. 39-52). Ámsterdam: John Benjamins.

Meara, P., y Jones, G. (1990). Eurocentres vocabulary size test. 10KA. Eurocentres.

Nation, I. S. P. (1990). Teaching and learning vocabulary. Nueva York: Newbury House.

Nation, I. S. P. (2001). Learning vocabulary in another language. Cambridge: Cambridge University Press.

Oxford, R., \& Crookall, D. (1990). Vocabulary learning: A critical analysis of techniques. TESL Canada Journal, 7, 9-30.

Paribakht, T. S., \& Wesche, M. B. (1993). Reading comprehension and second language development in a comprehension-based ESL program. TESL Canada Journal, 1(1), 9-27.

Paribakht, T. S., \& Wesche, M. B. (1999). Reading and "incidental" L2 vocabulary acquisition: An introspective study of lexical inferencing. Studies in Second Language Acquisition, 21(2), 195-224.

Pigada, M., \& Schmitt, N. (2006). Vocabulary acquisition from extensive reading: A case study. Reading in a Foreign Language, 18(1), 1-28.

Prince, P. (1996). Second language vocabulary learning: The role of context versus translations as a function of proficiency. Modern Language Journal, 80, 478-493.

Robinson, P. (2005). Cognitive complexity and task sequencing: A review of studies in a componential framework for second language task design. International Review of Applied Linguistics in Language Teaching, 43(1), 1-33.

San Mateo-Valdehíta, A. (2012). Aprendizaje de léxico en español como segunda lengual lengua extranjera: investigación sobre la efectividad de tres tipos de actividades para aprender vocabulario (Tesis doctoral), UNED. 
San Mateo-Valdehíta, A., \& Chacón-García, C. (2019). Learning word class in a second Language through vocabulary learning activities: Definition-choosing, gap-filling, and sentence-writing. Journal of Spanish Language Teaching, 6(1), 49-63.

Sánchez Griñán, A. (2008). Enseñanza y aprendizaje de español como lengua extranjera en China. Retos y posibilidades del enfoque comunicativo (Tesis doctoral), Universidad de Murcia.

Schmidt, R. (1993). Awareness and second language acquisition. Annual Review of Applied Linguistics, 13, 206-226.

Schmitt, N. (2000). Vocabulary in language teaching. Cambridge: Cambridge University Press.

Seibert, L. C. (1930). An experiment on the relative efficiency of studying French vocabulary in associated pairs versus studying French vocabulary in context. Journal of Educational Psychology, 21, 297-314.

Swan, M. (1997). The influence of the mother tongue on second language vocabulary acquistion and use. En N. Schmitt y M. Mc Carthy (Eds.), Vocabulary: Description, acquisition and pedagogy (pp. 156-180). Cambridge: Cambridge University Press.

Webb, S. (2005). Receptive and productive vocabulary learning: The effects of reading and writing on word knowledge. Studies in Second Language Acquisition, 27, 33-52.

Webb, S. (2007). Learning word pairs and glossed sentences: The effects of a single context on vocabulary knowledge. Language Teaching Research, 11(1), 63-81.

Webb, S. (2009). The effects of receptive and productive learning of word pairs on vocabulary knowledge. RELC Journal, 40(3), 360-376. 
Apéndice 1: Ejemplos de los métodos de presentación del vocabulario

\section{Lista}

Chochear: $v$. manifestar las debilidades propias de la vejez.

Jeta: $n$. coloq. cara.

Descojonarse: $v$. reírse mucho y con ganas.

Cerote: $n$. miedo.

Algaraza: $n$. ruido festivo.

Cachas: adj. coloq. musculoso

\section{Oraciones}

Chochear: $v$. manifestar las debilidades propias de la vejez.

Los efectos de la vejez son evidentes en su forma de hablar y comportarse, ya chochea.

Jeta: $n$. coloq. cara.

Durante el partido, el balón le dio en toda la jeta; hasta le sangró la nariz.

Descojonarse: $v$. reírse mucho y con ganas.

Cuando se enteren del ridículo que hice, mis amigos se descojonarán de mi seguro.

Cerote: $n$. miedo.

Las películas de terror me producen asco y cerote.

Algaraza: $n$. ruido festivo.

Se nota que son fiestas, la algaraza de la gente hace imposible dormir.

Cachas: adj. coloq. musculoso.

Desde que vas al gimnasio, te estás poniendo muy cachas.

\section{Texto glosado (mismo texto para el método de Inferencia, sin glosas ni resaltes)}

Hace un par de fin de semanas, varios amigos salimos de fiesta. Estábamos muy animados porque la semana había sido dura en el trabajo y por fin podíamos descansar y relajarnos después del tute que nos habíamos dado en la oficina. Cuando fui a la barra a pedir una copa, un viejo carca se me acercó y empezó a hablarme. Su actitud dejaba claro que la edad no lo estaba tratando bien, era evidente que chocheaba. De repente, me tocó el culo. Mi reacción fue golpearle la jeta con todas mis fuerzas. Me hice daño en la mano, pero a él le sangró la nariz. Un poco más tarde, varios chicos guapos y cachas entraron en el bar. Mi amiga, a quien le encanta fisgonear, no se pudo contener y se acercó a hablar con uno de ellos. Al rato, todos estábamos sentados en la misma mesa, aunque con la algaraza que había en el bar casi no se podía escuchar a quien teníamos enfrente.

Uno de los chicos era supergracioso y todos nos descojonábamos de sus chistes. Sin embargo, el resto de sus amigos había bebido demasiado y ese ambiente gracioso y de 
vacile se convirtió poco a poco en agresividad. Uno de ellos se enfadó sin razón alguna, parecía un amente, fuera de sí. Acusó a mi amiga de ser una sonsolilla porque, según él, ella solo hablaba con él para conseguir bebidas gratis. Justo en ese momento, ese mismo chico, a quien el alcohol había ajado el rostro con 40 años más, saltó encima del más enteco de nuestros amigos y empezó a golpearle salvajemente. Yo empecé a sentir mucho cerote porque mi amigo estaba tirado en el suelo, inmóvil, y pensé que quizá habría espichado.

Tute $n$. coloq. esfuerzo intenso.

Carca adj. coloq. retrógrado, de ideas antiguas.

Chochear $v$. manifestar las debilidades propias de la edad.

Jeta $n$. coloq. cara.

Cachas adj. coloq. musculoso.

Fisgonear $v$. curiosear, hacer lo necesario para enterarse de los asuntos de los demás.

Algaraza $n$. ruido festivo.

Descojonarse $v$. reírse mucho y con ganas.

Vacile $n$. coloq. tomadura de pelo.

Amente adj. loco.

Sonsolillo, -a adj. coloq. astuto y hábil en beneficio propio.

Ajar $v$. envejecer.

Enteco. -a adj. débil.

Cerote $n$. miedo.

Espichar v. coloq. morir.

\section{Apéndice 2: Ejemplos de las pruebas de evaluación}

\section{Recuperación de la forma}

A continuación tiene una serie de oraciones que debe completar con las palabras que ha visto en la tarea de presentación del vocabulario. Solo una opción es posible, por lo que una misma palabra no puede usarse para completar varias oraciones. Preste atención también a que quizá es necesario conjugar el verbo o escribir la palabra en el número (singular/plural) y género (masculino/femenino) adecuados. Si no lo sabe, deje el hueco sin rellenar.

- Mi vecina, pese a no ser demasiado mayor, tiene una mentalidad bastante en cuanto a los nuevos modelos de familia. 


\section{Recuperación del significado}

A continuación tiene una serie de oraciones para las que debe explicar su significado. Hágalo preferentemente en español. En caso de no ser capaz, puede hacerlo en su lengua materna. Si no lo sabe, no escriba nada.

- "Ella no para de fisgonear" significa que ella no para de

\section{Reconocimiento de la forma}

A continuación tiene una serie de oraciones que debe completar con algunas de las palabras que tiene más abajo. Solo una opción es posible, por lo que una misma palabra no puede usarse para completar varias oraciones, y habrá palabras que no deben usarse. Si no sabe la respuesta, no complete el espacio en blanco.

- Se consideraba muy feo, por lo que cuando le tocó la lotería lo primero que hizo fue operarse la

\begin{tabular}{|c|c|c|c|c|}
\hline chochear & sonsolillo,-a & algaraza & cachas & ajar \\
\hline vacile & carca & descojonarse & cerote & tute \\
\hline fisgonear & amente & jeta & espichar & enteco, $-\mathrm{a}$ \\
\hline
\end{tabular}

\section{Reconocimiento del significado}

A continuación tiene una serie de oraciones para las que debe explicar su significado. Marque la opción más adecuada de las alternativas propuestas. Solo una es posible. Si no sabe la respuesta, no marque ninguna opción.

- "Los perros me dan mucho cerote" significa que los perros me dan
a. compañía
b. alegría
c. alergia
d. miedo 